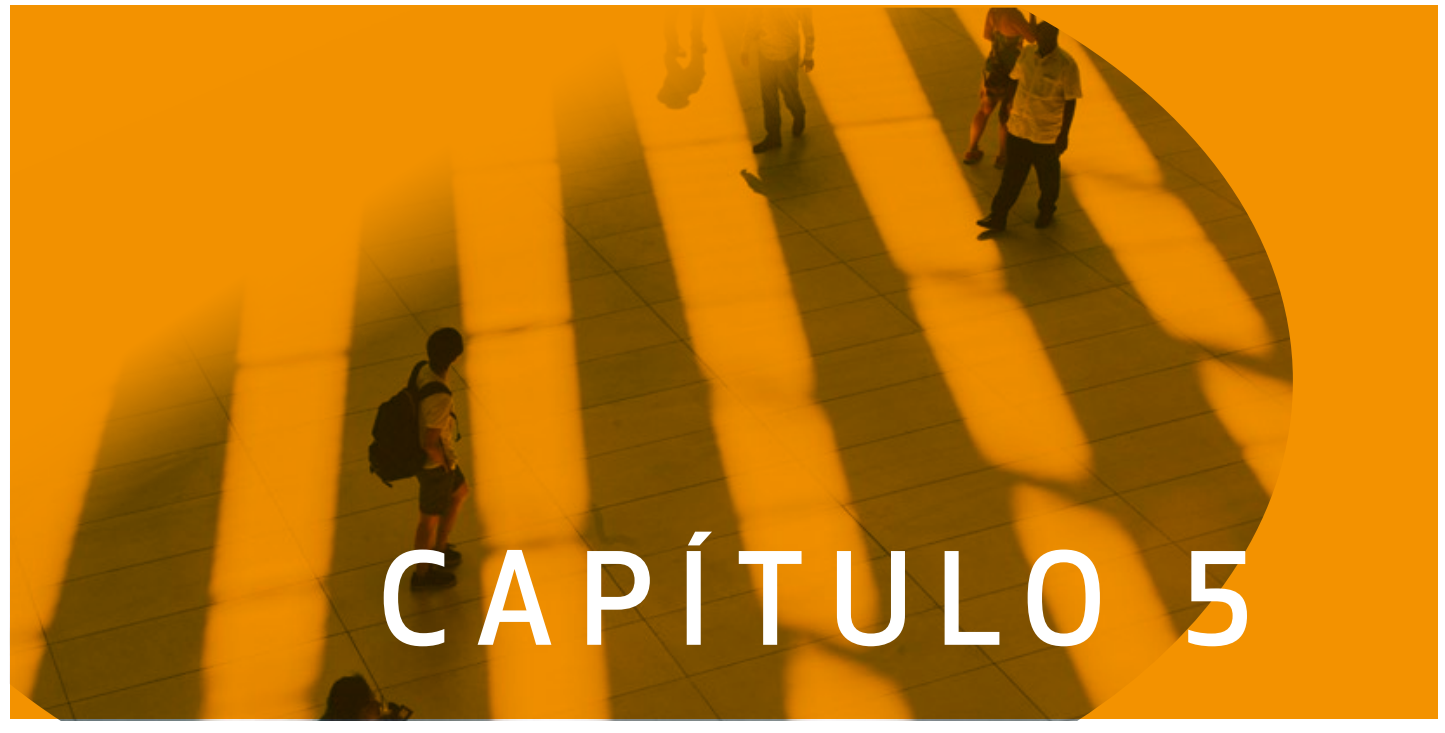





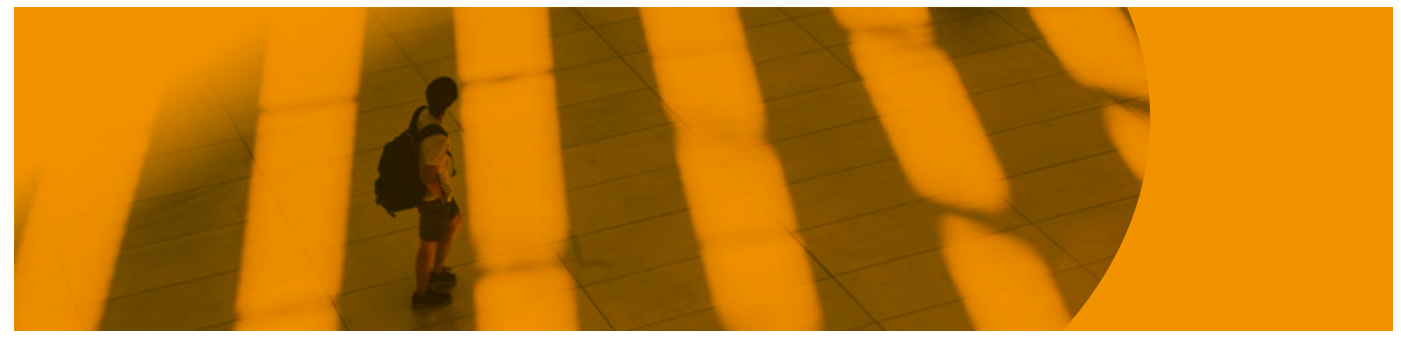

\section{Apuntes críticos sobre ética y responsabilidad social en contabilidad y finanzas}

\section{José Obdulio Cúrvelo Hassán}

Doctor en Contabilidad, Universidad de Valencia, España. Magíster en filosofía, Universidad Santo Tomás, Colombia. Contador público y especialista en revisoría fiscal y control de gestión de la Universidad Cooperativa de Colombia. Investigador asociado, dedicado a temas referidos a la contabilidad social, ambiental y de ética y responsabilidad social en los ámbitos del control de gestión. Profesor y líder del grupo de investigación Contabilidad y Entorno Social, de la facultad de contaduría pública, de la Universidad Cooperativa de Colombia, campus Bogotá.

Correo electrónico: jose.curvelo@ucc.edu.co

\section{Juan Pablo Cendales Rodríguez}

Contador público y Magíster en Administración por la Universidad Nacional de Colombia. Investigador dedicado a temas de control de gestión y responsabilidad social. Actualmente profesor e integrante del grupo de investigación Contabilidad y Entorno Social de la facultad de contaduría pública, de la Universidad Cooperativa de Colombia, campus Bogotá.

Correo electrónico: juan.cendalesr@campusucc.edu.co

\section{Martha Lucía Fuertes Díaz}

Doctora en Administración Gerencial (C), Universidad Benito Juárez, México. Magíster en Administración con énfasis en Finanzas Corporativas, Universidad ICESI, Colombia. Especialista en Finanzas con concentración en Finanzas Avanzadas, Universidad ICESI, Colombia. Contadora Pública, Universidad Santiago de Cali, Colombia. Docente Universidad Nacional Abierta y a Distancia, UNAD, Colombia. Investigadora principal Grupo de Investigación ilama.

Correo electrónico: martha.fuertes@unad.edu.co

ORCID: http://orcid.org/0000-0001-8468-1867

Google Scholar: https://scholar.google.com.co/citations?user=|P5s- 


\section{Introducción}

La gestión de la sostenibilidad en las organizaciones ha desembocado en desarrollos importantes en la contabilidad. De manera especial en el subsistema de información de control de gestión, conllevando a nuevas formas de comprender y entender la función social de las empresas junto con el reconocimiento a las formas de vida humanas. Lo anterior implica un suministro de información diferente, que posibilite en los diversos grupos de interés prácticas de control oportunas y pertinentes, de igual manera, el diseño de estrategias para obtener resultados empresariales armónicos junto con las responsabilidades asumidas en la conservación de la vida.

En este escenario, la contabilidad ha jugado un papel decisivo en la medida en que asume la responsabilidad de generar información amplia para la comprensión de la dimensión ética y de la responsabilidad social y ambiental de las organizaciones que informan. Siendo así, la contabilidad presenta un desarrollo importante en materia de captura y medición de dichos fenómenos, lo que da cuenta de un dinamismo de sus objetos de representación y en sus objetos de estudio.

Bajo este horizonte de sentido y producto del proyecto de investigación"Análisis situacional y prospectiva de la teoría e investigación en contabilidad", auspiciado por CONADI, se presentan unos apuntes críticos sobre ética y responsabilidad social en contabilidad y finanzas, los cuales se desarrollan en tres apartados.

El primer apartado busca avanzar hacia el carácter contractual de la contabilidad, siguiendo los postulados de la teoría de la contabilidad y el control de Sunder (2007). El segundo apartado explora las principales tradiciones éticas para generar algunas reflexiones sobre su noción contemporánea. El tercer apartado centra la atención en la responsabilidad social como una expresión de confianza pública y valores empresariales. De las reflexiones de los anteriores apartados se generan las reflexiones que a título de conclusión se traza el capítulo de esta obra denominada: "Oportunidades, necesidades y tendencias de la educación contable en Colombia". 


\section{El carácter contractual de la contabilidad}

Cuando se afirma que la contabilidad es decisiva en los contratos que se configuran en la sociedad contemporánea, además de afirmar que la contabilidad favorece las instituciones y el orden jurídico, se otorga una fuerte asociación con el control. Esto implica una multiplicidad de factores que deben ser dilucidados y encarados desde la amplitud misma del término. Frente a lo antes expuesto, la literatura da cuenta de una filosofía orientada a la inspección y vigilancia, y otra conformada por discursos desde sus fines de control, tanto formal como informalmente.

Lo antes expuesto evidencia que las manifestaciones del control tienen un fuerte arraigo cultural, entendiendo la cultura en los términos propuestos por Kattenbelt (2008), como la apreciación de los sujetos sobre el estado actual del concepto y propósito de la intermedialidad. En dicho contexto se puede leer y entender cuando Ocampo Salazar et al., (2009) plantean:

[...] De las diferentes épocas en las que se ha dividido la historia, la contemporánea, presenta una profusión de discursos sobre la realidad que debe representar la Contabilidad, mencionando que ésta no sólo capta aspectos económicos, sino que debe reflejar aspectos sociales, ambientales, culturales, entre otros. En este sentido se intenta hacer una crítica a la lectura que desde el enfoque financiero (reduccionista) se propone, de elementos como el capital intelectual, el capital ambiental, el patrimonio cultural, etc., que no implica el surgimiento de la Contabilidad Ambiental o de la Contabilidad Cultural -por hacer mención de algunas- sino que son nuevos aspectos informados y comunicados por la Contabilidad Financiera, pues la captación y la comprensión de estos fenómenos se está realizando desde este enfoque... La relación Contabilidady Cultura es un tema con poco avance teórico y práctico, razón por la cual empezar a elaborar algunos aportes, constituye un importante adelanto para aquellas personas (estudiantes, docentes, profesionales e investigadores) que quieran indagar sobre la temática (p. 219). 
Atendiendo que la contabilidad asume elementos como el capital intelectual, la gestión del conocimiento y el patrimonio cultural, se hace pertinente que cualquier rastreo que se haga sobre dicha noción tenga como supuesto la filosofía del control, su adscripción a procesos culturales que connotan la manera de hacer negocios y de establecer relaciones comerciales. De igual forma, a las manifestaciones y construcciones alrededor de la multiplicidad de grupos de interés. Este último aspecto, de modo particular, reviste una importancia significativa por cuanto la revelación contable, desde una perspectiva externa, así como los estilos de liderazgo y control, desde una perspectiva interna, son resultado de los grupos de interés con los que interactúa la empresa y que ejercen presión por las necesidades que de ellos se tiene (García, Gómez y de las Heras, 2018).

Esta afirmación cuenta con una literatura diversa e igualmente se reconoce una ideolología que la arraiga históricamente a la inspección y a la vigilancia, aspectos necesarios para el ser humano desde la superación del nomadismo. De igual manera, entraña la literatura una tradición cuya noción se adscribe al sistema económico moderno, cuyo principal referente se puede anclar en las concepciones de Hegel (2012), con su obra Los principios de la Filosofía del Derecho, cuando presenta una vía para el tránsito de la moral a la etnicidad:

El saber es, pues, no-ético y ético, existencial y sistemático. De ahí que el título de filosofía del derecho sea inadecuado, a no ser que se entienda como equivalente a la filosofía entera de la praxis (derecho, moralidad y eticidad) y al mundo ético histórico que le corresponde, que es la consumación de la modernidad. El punto de vista sistemático está ya implícito en los "principios", aun cuando en su sentido primeramente didáctico como líneas fundamentales (Grandlinien), dado su carácter de manual universitario (p. 3).

Si bien queda evidenciado el tránsito de la moral a la eticidad, deberá tenerse presente que la concepción propuesta en Hegel de vida racional, además de ser incondicionado es omniabarcante, se edifica y construye en la interacción entre la idea absoluta, lo práctico y lo teórico. Por consiguiente, hay un error 
lógico y epistemológico al limitar que las tradiciones del control en la contabilidad sean la latina y la anglosajona. La relación práctica que subyace en la información y el control contable es compleja y dialoga con el derecho, la moralidad y la ética.

En otras palabras, la relación con el derecho, la moralidad y la ética del control contable sitúa en la cúspide a la razón. Es decir, el control contable como una categoría con raíces sociológicas, que le hacen trascender al instrumentalismo de la tecné (Tancredi, 2009), la producción y reproducción de información en la empresa, y sin asidero en la vida social. Esta relación del derecho, la moralidad y la ética avanza a otros estadios cuando Hegel llama a superar el estado de naturaleza.

De superarse el estado natural, la función del control contable, tanto en términos abstractos, como en lo referente a la capacidad de atender las necesidades de la sociedad, se construye como un andamiaje de relaciones que se tejen y entretejen para garantizar la vivencia del derecho, la moralidad y la ética. Lo anterior, además de evidenciar la importancia de la contabilidad en la sociedad contemporánea, también devela que aún existen terrenos áridos, sin trasegar, alrededor de la teoría clásica del contrato social. Los cuales se han producido por la función social actual de la contabilidad, donde debe acercarse a otros lenguajes para conversar desde las investigaciones en contabilidad y finanzas, lenguajes más finos y delineados que contemplan lo político y lo social. Esta resulta ser una tarea tan necesaria como urgente. El lenguaje que emplea la contabilidad siempre ha sido una piedra angular para su función de control, por cuanto es la garantía de la representatividad de la realidad económica, reflejada en la información que se transmite a sus múltiples usuarios y que es soporte de su proceso de toma de decisiones. De la misma forma, los medios de comunicación de la información contable ejercen una influencia notoria (Requena, 1973; Macintosh y Baker, 2002).

Esta finura de las apuestas en investigación que contemplan lo político y social que subyace en la contabilidad, tienen importantes referentes en tradición, configurada por el iusnaturalismo, con lo cual se hace referencia a la teoría de 
la contabilidad y el control de Sunder (2007). Lo anterior, en la medida en que lo contractual en la contabilidad, como horizonte de sentido, va de la mano con los cambios que devienen tanto en el derecho como en la filosofía política. Así mismo, colindan con las lógicas del método de doble efecto de Lucas Paciolo al decir debe y haber, que es equivalente a deberes y derechos en la visión de Cerboni, categorías conceptuales que no tienen vida en el orden natural sino en las cambiantes leyes humanas. Igualmente, incluyen el pleno reconocimiento de la propiedad privada y las nociones alrededor del derecho de propiedad.

Las organizaciones tienen diferentes significados para cada agente. Las empresas comerciales, por ejemplo, son empleadores para aquellos que trabajan en ellas: clientes para los proveedores de bienes y servicios; proveedores para sus clientes; benefactores para los que reciben su caridad; inversiones para aquellos que intervienen en ellas; contribuyentes para el gobierno; una amenaza para la supervivencia de sus competidores; burocracias impersonales para los más pobres y pilares de la libre empresa para los doctrinarios. Las organizaciones son vistas de manera diversa como redes complejas de relaciones humanas, funciones de producción, jerarquía e incluso como cestos de basura. Éstas, por su parte, se precian de ser depósitos de cultura y civilización y, a la vez, motores de cambio. Son también ridiculizadas como fuentes de indecencia y como obstáculos para el progreso de la sociedad.

Para el propósito limitado de tratar de explicar la naturaleza de la contabilidad y el control en las organizaciones, en este capítulo, se hará uso de una vieja e interesante idea, apoyada por estudiosos como Cyert y March, Simon, Bernard y Rousseau: una organización es simplemente un conjunto de contratos entre los agentes. Los contratos son acuerdos mutuos, formales o informales, tanto el alquiler de un apartamento como una cita para almorzar con un amigo. Los agentes son indistintamente individuos u organizaciones. Se da por sentado que los agentes son racionales, no eligen conscientemente aquello que no les gusta (Sunder, 2007, p. 23-24).

Lo anterior tiene mucho más asidero si se tiene de presente que es precisamente durante el siglo xvı (Lopes de Sá, 2008) donde las reflexiones europeas, 
alrededor de la contabilidad, alcanzan un alto grado de profundidad al tiempo que sientan las bases científicas y los fundamentos disciplinares de la misma. Dejando instaurada la crítica a todos aquellos pensadores contables que solo se han limitado a plantear la supremacía entre la tradición anglosajona y la europea, y no han abierto la posibilidad para el desarrollo del conocimiento, en la medida en que se han establecido criterios para la sistematización y difusión del saber con una rigurosidad propia para este campo del conocimiento en otros paises.

Es de precisar que cuando se analiza ampliamente la teoría de la contabilidad y el control, se puede inferir que es la posición sobre iusnaturalista la que no ha sido reflexionada ampliamente en las diferentes investigaciones en contabilidad. En la medida en que la administración de los bienes no podría estar a atada a una supremacía de la razón divina sobre el derecho positivo. Es criticable también al iusnaturalismo soslayar la naturaleza clasista del derecho buscándole una raíz alejada del Estado (Tapia Sánchez, 1995; Masbernat, 2005), ello obedece al reconocimiento de una relación recíproca entre las partes contratantes, la cual no puede ser diluida en virtud de una razón superior. Esto es sensible al momento de tratar la riqueza generada en la medida en que, pese a que existe una utilidad producto de una inversión realizada por alguien, ese alguien no puede, amparado en ser el dueño del capital, desconocer que la misma proviene de una multiplicidad de factores que residen dentro de una sociedad a la cual él deberá retribuirle. Precisamente el reconocimiento de la participación de múltiples factores y actores en el quehacer organizacional, tanto externos como internos, es lo que ha demandado el perfeccionamiento de prácticas de rendición de cuentas (Accountability).

Bajo este contexto, lo que se plantea es que la premisa mayor dentro de la teoría de la contabilidad y el control, que no trató el maestro Sunder (2007), y el método de la partida doble, como práctica legítima en los negocios, responde a una oposición, a la forma de hacer transacciones comerciales reguladas por el poder divino. De allí deviene el tratamiento de la utilidad y el sistema financiero, por lo tanto, desde ella se erige una forma de operarlos, soportado en el derecho y centrado en el individuo como norma superior: 
El contractualismo no constituye una doctrina única y uniforme, sino más bien una teoría extremadamente adaptable a diferentes contextos -lo cual explica su vitalidad y capacidad de evolución- que surge de la disolución de la concepción teocrática del mundo y de la ruptura del pacto establecido entre razón y ley eterna, y el reemplazo de ésta por la razón natural. El triunfo de las ciencias de la naturaleza y de la filosofía mecánica asestaron finalmente el golpe de gracia a la concepción de la lex aeterna e hicieron posible su reemplazo por la idea del individuo como única realidad sustantiva en que apoyar la percepción del mundo. A partir de este momento se intenta concebir al ser humano por medio de la disección de la sociedad, descomponiéndola en fragmentos hasta alcanzar su unidad atómica: el individuo, para, desde ahí, recomponerla a través de leyes psicológicas que permitan explicar el comportamiento del propio individuo (Torrado, 2008).

Así, hay dos elementos que requieren ser develados: el primero es que hay una clara ruptura con la tradición del poder de sangre, la cual es sustituida por un poder centrado en el individuo; el segundo, que será tratada más concretamente por Adam Smith (1994) y David Ricardo (1959), establece un marco ético propio a los negocios, el cual guarda clara relación con lo que se ha venido dando hasta el presente y que posibilita entender de mejor manera la dimensión contractual de la empresa y el rol de la contabilidad en la sociedad como una herramienta de control entre los agentes, entendiendo la misma en múltiples vías, por una parte, con relación a contratantes con la sociedad y por la otra entre contratantes y el capital.

Lo anterior lleva, parafraseando a Hobbes (2013), a que se considere como única función de la contabilidad el control:

Cuando un hombre razona, no hace otra cosa sino concebir una suma total, por adición de partes; o concebir un residuo, por sustracción de una suma respecto a otra: lo cual — cuando se hace por medio de palabras - consiste en concebir a base de la conjugación de los nombres de todas las cosas, el nombre del conjunto: o de los nombres de conjunto, de una parte, el nombre de la otra parte. Y aunque en algunos 
casos — como en los números_, además de sumar y restar, los hombres practican las operaciones de multiplicar y dividir, no son sino la suma de cosas iguales, y la división o la sustracción de una cosa tantas veces como sea posible... y los políticos suman pactos, uno con otro, para establecer deberes humanos. En cualquier materia en que exista lugar para la adición y la sustracción, existe también lugar para la razón (pp. 57-58).

En este orden, los inversores están en peligro inminente con sus capitales y requieren un clima de seguridad, requieren, ante todo, de herramientas que le den cuenta de la manera como crece su capital, pero también requerirán de una estructura o de un aparato superior que garantice el seguir creciendo y la optimización de sus retornos, encontrando en la figura del Estado dicha posibilidad. Este requerirá de dinero para su operación en razón a lo cual también requerirá de la misma herramienta. Siendo así, se infiere el carácter contractual de la contabilidad y la función de la misma como herramienta de control, la cual es pertinente en el marco más amplio del pensamiento hegeliano, aun cuando llegue a tal afirmación solo por inferencia, en la medida en que él nunca hizo alusión a la contabilidad. En el texto de Amelia Valcárcel (1988) se plantea:

Enjuiciemos pues la negatividad en la que se ha resuelto la libertad absoluta. Y comienza. La voluntad universal no es positiva y, sin embargo, la pura autoconciencia es absoluta positividad; no se le escapa entonces que identificarse con una voluntad universal que la aniquila es un mal negocio. Sabe que «la voluntad universal es su puro saber y querer... el puro saber cómo esencia es la voluntad universal, pero esta esencia es simplemente el puro saber. La autoconciencia es, por tanto, el puro saber de la esencia como puro saber». Lo que, en resumidas cuentas, podría intentar traducirse, aun corriendo el riesgo de saber un poco iconoclasta, por: sabemos que la universalidad es una idea y una práctica que efectivamente se pueda llevar a cabo. Incluso tenemos noticia de la obligación de apuntalar una voluntad universal, pero no se nos escapa que sólo existe una teoría y que, en los hechos, en cuanto obra, es facción. También comprendemos, porque somos per- 
sonas mayores, que todo el asunto es bastante especulativo y que el querer puro no tiene contenidos, pero preferimos funcionar con estas especulaciones, que son solamente, bien lo sabemos, pensamientos. Todas estas evidencias nos conducen a un cierto desencanto sobre nuestras posibilidades. Desde ahora, guardamos nuestras casacas ilustradas porque las reglas del juego han sido abolidas. A nadie se le escapa lo frágil y ridículo de la voluntad general o lo que ocultaba. Vayamos apagando las luces, que se ha terminado el tiempo que la historia nos concedió para la representación. No hay voluntad general, no igualdad, no absoluta libertad. De la fraternidad no merece siquiera la pena tomarse el trabajo de hablar. De este desencanto, sale a la luz una nueva figura, la última que se tratará, la conciencia moral, más exactamente, el Espíritu Moral (p. 227).

Apoyados en Valcárcel, cuando cita a Hegel, puede afirmarse que la dimensión del control de la contabilidad es inherente a su contractilidad, la cual se da porque en el mercado participan individuos aislados con intereses particulares, quienes demandan garantías para mover libremente sus capitales. Dado que los mismos se adscriben a una sociedad que también pide respeto por el carácter común de los insumos requeridos para el normal desarrollo del proceso productivo.

\section{Principales tradiciones éticas para generar algunas reflexiones sobre su noción contemporánea}

Si partimos por considerar la ética como un texto susceptible de ser leído y comprendido con fragmentos y esquemas conceptuales, podría esperarse que dentro de ella existan contextos que derivan en una diversidad de significados. De ser así, los valores axiológicos no son su única función social. El término valor que se ha usado debe precisarse dado que, mientras en la economía capitalista está asociado al mercado, en la axiología está referido a los valores morales. Pese 
a que cada una de estas nociones de valor tiene una multiplicidad de diferencias, encuentran un punto de confluencia en la esfera de lo público: la vida en sociedad. Por consiguiente, la ética no solo obedece a las relaciones con otros semejantes sino a la interacción con otras formas de vida, que va desde la espontaneidad de las vivencias hasta las obligaciones con el Estado y la sociedad.

En lo relacionado con su ontología histórica, puede afirmarse que la ética proviene de una marcada tradición que la relaciona a las costumbres: modo habitual de obrar. En su momento, Zenón (Leningrad, 1988) la consideró como fuente de vida de la que emanan los actos humanos. Se establece una de las primeras diferencias al confrontarla con Aristóteles, quien la relaciona en términos de formas de ser o carácter"de un modo más amplio como las disposiciones del hombre en la vida, sus hábitos, sus costumbres modo o forma de vida" (p. 56).

Desde esta noción propuesta por Aristóteles se deduce, en términos de bienes, tres grandes tipos, a saber, "los exteriores, los del cuerpo y los del alma" (Domínguez, 2006). Se relaciona la ética con la felicidad y ésta a su vez con una cualidad de la persona que depende de su propia voluntad. En este orden de ideas, y a la luz de los planteamientos formulados, cabe preguntarse ila felicidad se aprende o se lleva consigo como si fuera una segunda naturaleza?, o por el contrario, ¿proviene la felicidad de alguna divinidad? (Kant, 1997).

Es cierto que Aristóteles no hace referencia a los humanos, por ello la ética se contextualiza en la cultura y no precisamente como la conciencia humana sino como el saber ser feliz, hecho que demanda conocerse y conocer lo que afecta al individuo y le impide conquistar la felicidad (Aristóteles, 1981; Colín y Montoya, 1986; Tejedor, 1991; Abad, 1995). Este referente es desafiado con la postura de ética de Spinoza (2003) quien la lleva a nivel de tratado, como un saber organizado, tranquilizador, un saber armado. Como si fuera una ciencia, y busca explicar el nacimiento o genealogía a las distintas valoraciones que le dan los afectos. Lo que se da es una sustitución de las acciones asociadas a la ética por afectos. 
En términos de este capítulo, se toman como referencia dos grandes maneras de entender la ética: Ia discusión Nicomaquea y la ética de Spinoza. Con el objetivo de reflexionar sobre la perspectiva de ella en contaduría, se ampliará el espectro de manera que permita entender el papel contable en los cambios de las redes sociales, por una parte; y por la otra, las tendencias de las organizaciones y las exigencias de información. En gran medida consideramos que Foucault, en su obra dedicada a la sexualidad, nos podría dar luces al respecto.

Michel Foucault proponeuna ética en términos de los procesos de subjetivación de los sujetos modernos. Es decir, la crítica a los procesos de subjetivación histórica, de donde se devienen la voluntad del saber y la preocupación por sí mismo (Foucault, 1987). La ética está dada con relación al sujeto y no a la estructura. En esta noción de ética, Foucault posibilita la reflexión sobre problemas netamente éticos. La ética es entendida para él en la relación afectoplacer (Foucault, 1987). Ahora, si se habla de los procesos de subjetivación de los sujetos modernos, la ética es la única posibilidad que tiene el ser humano de pensarse y ser pensado, de cuestionar su existencia, lo que equivale a decir que es un desprendimiento del sujeto, de su forma, y de inquietarse por la búsqueda de sí mismo (Quiceno, 2003). Estos planteamientos son diferentes a la tradición que hemos heredado y que nos hace leerla y entenderla como un ideal de conducta en término de fines y medios. Esto para Foucault constituye una forma de violencia institucionalizada que imposibilita que las personas sean capaces de pensar su propia existencia; lo cual se refiere a la moral y no a la ética.

Foucault afirma, con relación a la moral, que es un código que prescribe las conductas y las formas de relaciones humanas. Por consiguiente, es una especie de listado donde se relaciona lo que está bien y lo que no lo está; lo que debe ser premiado y lo que debe ser sancionado. Están estrechamente relacionados a la forma de desarrollar ciertos actos humanos. Lo anterior es diferente a la ética en tanto ella es la manera en que cada sujeto se inquieta por sí mismo en calidad de agente moral. La ética se ocupa no del juicio de la actuación, como buena o mala, sino de la reflexión que emerge del sujeto y de sus actos. Esta 
noción de ética implica los hábitos y las costumbres, los cuales proceden del sujeto y no de los códigos.

En términos de conclusiones y, a la luz de Foucault, la ética está vinculada a las prácticas del yo. Es, según como se piensa el yo, de donde se derivan las formas de la ética. En este orden de ideas, cabe formularse las siguientes preguntas: ¿existe un lazo efectivo entre la información contable y los niveles de bienestar de la sociedad?, en caso de que exista ¿cuál es la relación entre la ética del contador y los actos de los sujetos en la sociedad? Estos interrogantes se intentarán abordar - sin que impliquen ser resueltos- en el apartado siguiente, el cual analiza las formas de aprensión de la ética en la contaduría.

\section{La responsabilidad social como una expresión de confianza pública y valores empresariales}

La teoría sobre la cual se posiciona el discurso de esta investigación es la de la contabilidad y el control, que establece una relación inherente entre ellas dado su carácter contractual. Este hecho es generado porque en el mercado participan individuos aislados con intereses particulares, quienes demandan garantías para mover libremente sus capitales. No obstante, dado que ellos se adscriben a una sociedad que pide respeto al carácter común de los bienes requeridos en el proceso productivo o prestación de servicios, terminan por delegarle a la contabilidad un rol como conciencia o espíritu moral, es decir, un sistema de contabilidad se diseña de manera eficiente para proporcionar información acerca del cumplimiento de los contratos por parte de sus agentes (Sunder, 2007).

Así mismo, propone Sunder que cada organización tiene un significado diferente configurado por el control en la organización y el control de la organización. En relación a los referentes filosóficos inherentes a esta teoría, se identifican: la crítica a la manera de concebir la suma total por adición de partes que ha 
tratado Hobbes (1651), el contrato social de Rousseau (1762) y la exterioridad determinante de la idea de Hegel (1807).

Dado los postulados de la teoría de contabilidad y el control, y las hipótesis de la investigación, se ha utilizado el enfoque normativo de la teoría de los stakeholders (Donaldson y Preston, 1995). Esta teoría plantea que los derechos de los grupos de interés son inherentes a ellos, por consiguiente, deben respetarse independientemente del beneficio o perjuicio que se genere. Siendo así, se afirma, en el marco de la investigación, que el estudio del impacto de una política de sostenibilidad deber ser leída y entendida más allá de la función social de la empresa e incluir, también, los intereses reales de los diferentes grupos. Al fin y al cabo, esta concepción es la que favorece un carácter más social para la contabilidad, en oposición al tradicional carácter funcionalista que privilegia el interés de algunos grupos sobre otros.

El segundo criterio que se ha tenido en cuenta es el diseño desde la red teórica, mediante la aplicación de la ruta propuesta por Ryan et al. (2004), para la construcción del estado de la cuestión. Esto implica: 1) escoger dos revistas especializadas que traten el problema de la investigación, siendo el criterio de selección que logren una sistematización significativa de la literatura y que cuenten con índice de citación e impacto, con la pretensión de identificar autores y trabajos empíricos; 2) de la revisión de la literatura se seleccionan algunos artículos publicados en un tiempo relativamente corto; 3) posteriormente se realiza un horizonte temporal y se establecen interconexiones según los intereses de la investigación, la que fue complementada, para mayor fiabilidad, usando el diseño teórico o bibliográfico (Hernández-Sampieri, et al., 2003; Ander-Egg, 2006; Sabino, 2007).

El tercer criterio es que la literatura seleccionada diserte sobre la existencia de la relación entre la responsabilidad social (RS) y ambiental y el comportamiento financiero de las empresas, conocido como la dimensión de la sostenibilidad empresarial (Elkington, 1994). Esto implica la contribución al desarrollo sostenible mediante la entrega al mismo tiempo de beneficios económicos, sociales y ambientales, lo que para Hart y Milstein (2003) se refiere a la creación 
de valor de la empresa en el nivel de estrategias y de prácticas que contribuyen a su continuidad en el tiempo.

Hay tres grandes concepciones sobre este planteamiento que requieren ser estudiadas. La primera de ellas, aunque las compañías declaran una relativa independencia entre estos aspectos la evidencia empírica lleva a afirmar lo contrario con el agravante que su discurso ético no favorece, es la sostenibilidad de los recursos (Cochran y Wood, 1984). La segunda es la relación entre política de responsabilidad y resultados financieros, la cual es positiva y significativa y además el índice ponderado por la RSE no tiene el mismo comportamiento cuando se estudia de manera independiente (Jong-Seo, et al., 2010), lo que está en consonancia con el concepto de grupos de interés aportado por Freeman $(1976 ; 1984)$ "cualquier grupo o individuo que puede afectar o que es afectado por el logro de los objetivos de la organización" (p. 24). La tercera proviene del análisis de las estructuras de las empresas, que plantea mayores rendimientos cuando la propiedad y el control de la empresa están alineados, con lo que se afirma que dichas estrategias favorecen el ingreso a sectores del mercado con preferencias y ratifican su legitimidad donde cuenta con cierto reconocimiento (Daily, 1992).

Finalmente, se entenderá como grupos de interés de una empresa, los individuos y los colectivos que contribuyen voluntaria o involuntariamente a su capacidad y a sus actividades de creación y generación de riquezas y que, por lo tanto, son sus potenciales beneficiarios y portadores del riesgo (Post, Preston y Sachs, 2002). Así mismo, se considera la empresa como una organización socioeconómica formada para crear riqueza para los múltiples colectivos que la componen (Olcese, et al., 2008). En cuanto a la difusión de prácticas de sostenibilidad, se consideran como la participación constructiva de las partes interesadas para aumentar la confianza externa en sus intenciones, actividades y en mejora de su reputación corporativa (Elkington: 1998).

Teniendo como horizonte de sentido las lecturas que se realizan sobrela relación entre la RS y ambiental y el comportamiento financiero de las empresas, el meta-análisis de Orlitzky, et al. (2003); Mahoney y Roberts (2007); Zhang y 
Stern (2007) afirman que dicho comportamiento ha tenido una tradición histórica, lo que se corrobora por Jones y Wicks (1999); Donaldson (1999); McWilliams y Siegel (2000, 2001); quienes adicionan que la empresa tiene el mismo comportamiento en todos los sectores y en distintos contextos. Nicolau (2008) afirma que cuando la empresa está contribuyendo a mejorar su entorno, satisface las expectativas de sus stakeholders e incrementa la riqueza, siendo así, este distintivo mejora el desempeño financiero y el valor de la empresa en el mercado, por lo tanto "los inversionistas consideran que esta empresa se esfuerza por tener un futuro sostenible y premian esta actividad con rendimientos anormales" (p. 992), no obstante, es necesario que la identidad e intereses estén acordes con la nacionalidad y al contexto en el que se desee impactar (Matten y Moon, 2008).

En este orden de ideas, los inversionistas están atentos a la gestión de la sostenibilidad empresarial y prefieren depositar su confianza en compañías que, desde su gestión, generan respuestas a las causas sociales y ambientales (Mahoney y Roberts, 2007). Este proceso implica la optimización de los recursos usados dentro del proceso productivo, de hecho, Orlitzky et al., (2003) comprueba que CSP se correlaciona positivamente con PPC y tiende a ser bidireccional y simultánea. En consecuencia, los mecanismos coercitivos del Estado, bajo el lema de garantizar el bienestar público y la sostenibilidad ecológica, terminan por legitimar la liberalidad de la actividad económica, lo que es cuestionado dado que para muchos estudiosos la función del Estado es dar prelación a la atención de las reclamaciones legítimas de la población civil en pro de su calidad de vida.

A este respecto, Tsoutsoura (2004) alude que la arquitectura de las empresas no está en consonancia con la función sustantiva del Estado, afirma que la responsabilidad social de aquéllas está centrada en ganar posicionamiento en el mercado; y, su desempeño socioambiental, se soporta en la eficiencia, que se mide por la optimización del uso de los recursos y los residuos, como categorías para aumentar sus desempeños económico-financieros (Spicer, 1978; Porter y Van Der Linde, 1995). 
Siendo así, cuando la RSE suple las funciones sustantivas del Estado se genera inequidad en la distribución de los recursos, aspecto sensible si se tiene en cuenta que la entrada en vigor de los derechos humanos económicos, sociales y culturales (OHCHR, 1996), terminó por ratificar el poder de las transnacionales sobre los recursos naturales. Esto se constata en normas complementarias que asignaron a la Organización Mundial del Comercio (OMC, 2010) la potestad de dirimir los conflictos relacionados con los recursos naturales y quien, a su vez, propuso el liderazgo empresarial como el camino a seguir para una mejor gobernanza mundial.

Estos hechos evidencian una tendencia hacia la construcción de un concepto de negocio a partir de discursos centrados en un modelo de rentabilidad, donde la transparencia es la base para la toma de decisiones. Así mismo, la situación y posición de sus trabajadores, el rol de los líderes de opinión, la academia, el medioambiente, las políticas públicas y los problemas sociales, son categorías para potenciar su desarrollo (Adams y Zutshi, 2004; King, 2002; Corregan y Cantú, 2005). Lo antes disertado lleva a firmar que la adopción de estrategias relacionadas con la RSC supone un cambio en la cultura organizacional y en los procesos operativos de la empresa (Joyner y Payne, 2002). Dicha política de sostenibilidad favorece su legitimidad (Márquez y Fombrun, 2005; Gardberg y Fombrun, 2002) con relación a la reputación sigue siendo medida como un criterio de RSE que se centra en el desarrollo de la comunicación creativa y asertiva (Fombrun, 2001).

Por otra parte, se observa que una de las estrategias de mayor impacto sigue siendo la divulgación de información relacionada con la inclusión social. Margolis y Walsh (2003), mediante estudios empíricos, documentan y sistematizan las iniciativas sociales más significativas de los negocios desde la década de los años setenta hasta finales del siglo pasado. Concluyen que las empresas incluyen, a título de RSE, sus aportes a la solución de problemas profundamente arraigados de la miseria humana, para maximizar sus riquezas, planteamiento que terminó por generalizarse con el diseño de políticas y sistemas de gestión denominada la triple bottom line (Carroll, 1999) 
que, como lo precisa McWilliams y Siegel (2001), constituye una estrategia de diferenciación con incidencia en la innovación de productos o procesos.

Acorde con lo anterior queda de manifiesto que la economía ha tenido un viraje significativo en la manera de concebir la relación entre la empresa y la sociedad, así como la instrumentalización de la teoría de los stakeholders a partir de la masificación de la triple bottom line. Frente a este fenómeno, JongSeo et al. (2010) propone un enfoque alternativo que favorezca controlar las tensiones entre los objetivos sociales y los económicos de las empresas desde una perspectiva sistemática. No obstante, Chang, et al. (2012) proponen que esto solo es viable por la necesidad que tienen las empresas de legitimarse en el mercado, lo cual implicaría, a juicio nuestro, responder a las expectativas de los consumidores sobre el cuidado de salud de sus familias, la calidad de la educación de sus hijos y una alimentación sana.

Esta situación da paso a que se incluya en el discurso empresarial la reflexión tanto del fin como de los medios que se fijan en sus actividades, además, dimensionar las consecuencias positivas y negativas de su praxis. Es en este contexto donde Krishnan (2007), mediantetrabajos experimentales, encuentra relaciones entre la RSE y la ética de los negocios, se complementa con Cox et al. (2008) al incluir variables relacionadas con el desempeño organizacional, y ambos concluyen que hay una marcada corriente a la que le inquieta la relación entre la manifestación del concepto y la percepción de las prácticas de RSE.

Estos planteamientos tienen un entramado histórico dado que Marrewijk en 2003 demostró que la ruptura entre RS y sostenibilidad de la empresa no es posible, pues en el mercado actual ambas confluyen en escenarios de intereses comunes, planteamientoquesecontraponea los postuladosclásicos(Freedman, 1962; 1970; 1984; Harrison y Freeeman, 1999). De ser así, resulta legítimo instaurar la crítica al Pacto Mundial de las Naciones Unidas (2000); la declaración de Río sobre Ambiente y Desarrollo (1992); los postulados de la Organización para la Cooperación y el Desarrollo Económico (OCDE, 2003; 2004). Incluso propuestas más heterodoxas como la de Amartya Sen (2000) que dan prelación a la eficacia en la distribución de los rendimientos en el colectivo de una sociedad. 
Lo que se evidencia en Marrewijk (2003) es una oposición a los esquemas de valores globales y, aunque tácitamente no se refiere al modelo del GRI, tampoco sale a su defensa, parecería que en él hay una marcada adhesión a las posturas de la sociedad civil global. En esta línea, Montiel (2008) plantea que los informes de sostenibilidad han terminado por desconocer los factores culturales con el agravante de que las trasnacionales suelen reportar y aunar sus mayores acciones en su lugar de origen, adicionándole que para O'Dwyer (2003); O'Dwyer, Owen y Unerman (2011) el modelo del GRI da supremacía al conocimiento financiero, la transformación en la gestión y a la constitución de la empresa, y no a su gestión de responsabilidad social y ambiental.

Con estos planteamientos, Peloza (2011) intenta superar los estudios de Griffin y Mahon (1997) que plantean que el uso a priori de las medidas de la CSP/ PPC podrían generar afectaciones significativas en la relación gestión social y ambiental de la empresa y su comportamiento financiero. En la medida en que la lógica financiera termina por condicionar la política de sostenibilidad, planteamientos que van en contraposición con Sarah y Peter Stanwick (1998), quienes encuentran que el rendimiento social de la empresa es afectado por su tamaño y su rentabilidad, lo que a su vez está estrechamente relacionado con la cantidad de emisiones contaminantes.

Peloza (2011) también instaura la crítica al uso del precio de las acciones y las medidas de contabilidad, como el retorno sobre el capital, como mecanismos de medición del impacto de dicha gestión, dando prelación a las herramientas de control en contextos particulares. Esto lo ratifican Park y Lee (2010) al proponer el conocimiento del escenario local como categoría complementaria a la transparencia y al fortalecimiento de los canales de comunicación con los stakeholders, con lo cual la identidad y el interés de las políticas de sostenibilidad estaría acorde con la nacionalidad y el contexto del cual se informa.

De igual forma, Moon y Matten (2008); y Deegan y Newson (2002) parten del análisis de los discursos de las empresas, y concluyen que se debe partir de la relación entre el medio ambiente y los recursos comprometidos para sostener una calidad de vida con holgura, así como el reconocimiento por parte de las 
industrias de las diferencias culturales y el compromiso con su entorno (Amato y Amato, 2011). A este respecto, hay una tendencia a enfatizar en las memorias de sostenibilidad sobre el impacto del despido de personal, el remplazo de trabajos de humanos por máquinas, y la masificación de la tecnología en la producción, sobre el buen nombre de la compañía y a sus resultados financieros. Diedrich y Bergström (2011) encuentran que una empresa puede asumir un papel activo en la construcción de la misma red de actores que demandan respuestas al impacto de sus actividades económicas e imponer a los demás actores su propio concepto y significancia de ser socialmente responsable, sin que ello altere su legitimidad.

Dichas situaciones han favorecido que, en la presente década, hayan proliferado estudios que verifican el resultado financiero de las compañías cuando la RS implica atender a valores éticos, el cumplimiento de normativas y criterios heurísticos como mecanismos de participación (Canela et al., 2011). Así mismo, lo evidencian estudios sobre legitimación de la actividad empresarial donde ciertos agentes desarrollan acciones que deprimen la calidad de vida y el bienestar de la población y de los recursos naturales (Chiu y Sharfman, 2011); lo mismo que el cuestionamiento al uso de un modelo único de reporte de memorias de sostenibilidad, al considerar que desconocen el contexto local (Aguinis y Glavas, 2012), y se retoman los planteamientos de Montiel (2008) agregándole la necesidad de incluir horizontes metodológicos que favorezcan un análisis diferencial y sus relaciones con la información que se captura desde la contabilidad.

\section{Conclusiones}

La revisión realizada permite afirmar que la contabilidad se considera en la sociedad contemporánea como el álgebra moral (Maas, 2016). Atendiendo la propuesta de Franklin, tratada por Mathews (1976), que reconocen los diversos propósitos o inclinaciones respecto de la toma de decisiones en situación. Lo anterior deja en evidencia la necesidad de abordar estos fenómenos desde y hacia la contabilidad, en tanto ella constituye el 
lenguaje de los negocios, conllevando, según la noción de la ética desde la filosofía analítica (Wittgenstein, 1965), a ser garante de los acuerdos de una comunidad lingüística.

Es igualmente evidente que la noción de responsabilidad social y su relacionamiento con la contabilidad conllevan a dilucidar los problemas morales relacionados con la corrupción y la manipulación de la información financiera, ello supone mejorar en transparencia y aportes a la construcción de la justicia social. Existe, en dicho contexto, un gran reto a los códigos de ética de tal forman que se diluciden los problemas de moralidad en las prácticas de manipulación de información financiera, de corrupción y las garantías de no repetición. Lo anterior es imperativo cuando se busca dar cumplimiento a las necesidades de los distintos usuarios de la información contable, en un propósito claro de no dar continuidad a estructuras de poder tradicionales en una economía de mercado, sino permitir la anhelada neutralidad de la contabilidad.

Finalmente, en materia de responsabilidad social, el nuevo esquema del GRI (2014), la promulgación de la ISO 26000 (2013) y la normativa global en materia de conservación ambiental (2013; 2014), han terminado por darle prelación en el informe a los derechos humanos, atendiendo a los enfoques y directrices de la OCDE (2004) y la declaración de la Organización Internacional del Trabajo (OIT, 2010). Esta situación ha generado nuevas investigaciones, tanto para establecer el impacto financiero en caso de no cumplir los compromisos de los contrayentes, como acerca del impacto que genera su cumplimiento (Kwong, et al., 2012). Puede decirse, en consonancia con Catherine M. Daily (1998), que en el campo de la gestión empresarial se requiere avanzar en el conocimiento de la eficacia de la RS y ambiental pero también en el diseño de modelos y teorías que con un enfoque diferencial rescaten características culturales, que redunden en una comprensión amplia de las estructuras, mecanismos de gobernabilidad y de sostenibilidad de recursos y de formas de vida humanas y no humanas. 


\section{Referencias}

Abad Pascal, J. (1995). La vida moral y la reflexión ética. España: Editorial McGraw-Hill.

Appelqvist, H. (2013). Ethics and aesthetics are one and the same? Wittgenstein's Tractatus: History and Interpretation, 40.

Appelqvist, Ö. (2013). The Political Economy of Gunnar Myrdal: Transcending Dilemmas Post-2008. Routledge.

Aristóteles. (1981). A poética clássica. Cultrix/EDUSP.

Benjamin Franklin (1972). Benjamin Franklin's technique of moral algebra as outlined in his letter of 19 September, 1772. Recovered from: https://www.researchgate.net/ profile/Ralph_Keeney/publication/13119982_Even_Swaps_A_Rational_Method_for_ Making_Trade-offs/links/02e7e52f52123c0af0000000.pdf

Boyce, G., and Greer, S. (2013). More than imagination: Making social and critical accounting real. Critical Perspectives on Accounting, 24(2), 105-112.

Brenner, S. N. (1992). Ethics programs and their dimensions. Journal of Business Ethics, 11(5-6), 391-399.

Brown, J., and Dillard, J. (2013). Critical accounting and communicative action: On the limits of consensual deliberation. Critical Perspectives on Accounting, 24(3), 176-190.

Brown, J., Dillard, J., and Hopper, T. (2015). Accounting, accountants and accountability regimes in pluralistic societies: taking multiple perspectives seriously. Accounting, Auditing \& Accountability Journal, 28(5), 626-650.

Chiapello, E. (2016). Critical accounting research and neoliberalism. Critical Perspectives on Accounting. 
Colin, J. Montoya J. (1986). Aristóteles: sabiduría y felicidad. Madrid, España: Editorial Cincel.

Dalton, D. R., Daily, C. M., Ellstrand, A. E., and Johnson, J. L. (1998). Meta-analytic reviews of board composition, leadership structure, and financial performance. Strategic management journal, 19(3), 269-290.

De Elea (s.f.).Biografía de Zenón. Volver a la geometría cuántica.

De Sa, A. L. (2008). Fundamentos da Contabilidade Geral-Com Referências Relativas à Reforma das Sociedades por Ações-Lei. Revista e Atualizada. Jurua Editora.

Domínguez García, V. J. (2003). El miedo en Aristóteles. Psicothema.

Donaldson, T., and Preston, L. E. (1995). The stakeholders theory of the corporation: Concepts, evidence, and implications. Academy of management Review, 20(1), 65-91.

Driessens, O. (2013). The celebritization of society and culture: Understanding the structural dynamics of celebrity culture. International journal of cultural studies, 16(6), 641-657.

Eberlein, B., Abbott, K. W., Black, J., Meidinger, E., and Wood, S. (2014). Transnational business governance interactions: Conceptualization and framework for analysis. Regulation \& Governance, 8(1), 1-21.

Fontrodona, J. (1999). Marketing social. ROA, JF Ética del marketing. Madrid: Unión Editorial.

Foucault, M. (1987). El uso de los placeres. Madrid: Editorial siglo XXI.

Foucault, M. (1987). La hermenéutica del Sujeto. Madrid: Editorial siglo XXI.

Foucault, M. (1987). La inquietud de sí. Madrid: Editorial siglo XXI. 
García, B., Gómez, J. and de las Heras, E. (2018). Debt pressure and interactive use of control systems: Effects on cost of debt. Management Accounting Research, 40, 27-46.

González, T. F., and Guillen, M. (2008). Organizational commitment: A proposal for a wider ethical conceptualization of 'normative commitment'. Journal of Business Ethics, 78(3), 401-414.

Hegel, G. W. F. (1988). Principios de la Filosofía del Derecho. Barcelona: Edhasa.

Hegel, G. W. F. (2012). Principios de la filosofía del derecho. Sudamericana.

Hegel, G. W. F., Gaos, J., y Ortega y Gasset, J. (2008). Lecciones sobre la filosofía de la historia universal. Alianza.

Hobbes, T. (2013). Del ciudadano y Leviatán. Tecnos.

Kant, I. (1997). What is enlightenment?

Kattenbelt, C. (2008). Intermediality in theatre and performance: Definitions, perceptions and medial relationships. Cultura, lenguaje y representación: revista de estudios culturales de la Universitat Jaume 1, 6, 19-29.

Maas, H. (2016). Letts Calculate: Moral Accounting in the Victorian Period. History of Political Economy, 48(1), 16-43.

Macintosh, N. and Baker, C. (2002). A literary theory perspective on accounting. Towards heteroglossic accounting reports. Accounting, Auditing \& Accountability Journal, 15(2), 184-222.

Marx, K. (1985). La Miseria de la Filosofía. Madrid: Sarpe.

Masbernat, P. (2005). Autonomía individual y autonomía colectiva como Fuente del Derecho. Revista de Derecho-Universidad Católica del Norte, 12(1), 99-120. 
Mathews, W. M. (1975). A critique of traditional drug education programs. Journal of Drug Education, 5(1), 57-64.

Mathews, W. M. (1976). Benjamin Franklin: A Father of Decision Making, Too. The Phi Delta Kappan, 58(1), 52-52.

McIntyre, D. D., Chen, C. and Jones, K. T., (2005). A reexamination of the factors important to selection of accounting as a major. Accounting and the Public Interest, 5(1), 14-31.

Murphy, P. E. (1995). Corporate ethics statements: Current status and future prospects. Journal of Business Ethics, 14(9), 727-740.

Passetti, E., Bianchi, L., Battaglia, M., and Frey, M. (2017) When Democratic Principles are not Enough: Tensions and Temporalities of Dialogic Stakeholders Engagement. Journal of Business Ethics, 1-18.

Peloza, J., and Shang, J. (2011). How can corporate social responsibility activities create value for stakeholders? A systematic review. Journal of the academy of Marketing Science, 39(1), 117-135.

Quiceno Castrillón, H. (2003). Curso de Epistemología, pedagogía y ética. Serie pedagogía y cultura.

Requena, J. (1973). Entes reales y entes representativos: medición e información. En La homogenización de magnitudes en la ciencia de la. España: Universidad Autónoma de Madrid.

Requena-Criado, M. (2008). El Contrato Social en el pensamiento de Hegel y de los filósofos contractualistas. Espéculo. Revista de estudios literarios. Universidad Complutense de Madrid. Recuperado de: http://www.ucm.es/info/especulo/ numero39/contsoci.html

Ricardo, D. (1959). Principios de economía política y tributación (No. Ae0450). FCE. 
Salazar, C. A. O., Rodríguez, C. L., y Martínez, J. C. G. (2009). Algunas relaciones entre contabilidad y cultura. Contaduría Universidad de Antioquia, (52), 215-258.

Sánchez, J. A. (1998). Transformaciones de la Constitución en el siglo XX. Revista de estudios políticos, (100), 57-100.

Smith, A. (1994). Riqueza de las naciones (1776). Madrid: Alianza, 37, 67-72.

Spinoza (2003). Book of Life: Freedom and Redemption in the Ethics. New Haven and London: Yale University Press.

Tancredi, E. (1999). El principio de responsabilidad de Hans Jonas: bases para una nueva ética frente al cambio ambiental global. Anuario de la División Geografía. Dpto. de Ciencias Sociales, UNLU.

Tapia-García, M., Yáñez-Arancibia, A., Sánchez-Gil, P., and García-Abad, M. C. (1995). Distribución, abundancia y reproducción de Priacanthus arenatus Cuvier (Pisces: Priacanthidae), en la plataforma continental del sur del Golfo de México. Biotropica, 232-237.

Tejedor Campomanes, C. (1991). Historia de la Filosofía en su marco cultural. España: Ediciones SM.

Tilghman, B. R. (1991). Ethics and Aesthetics in the Tractatus. In Wittgenstein, Ethics and Aesthetics. Palgrave Macmillan UK.

Tilghman, B. R. (1991). Wittgenstein, ethics, and aesthetics: The view from eternity. SUNY Press.

Tregidga, H., Milne, M. J., and Kearins, K. (2015). Ramping Up Resistance Corporate Sustainable Development and Academic Research. Business \& Society. doi: 0007650315611459. 
Valcárcel, A., Valcárcel, A., y de Quirós, B. (1988). Hegel y la ética: sobre la superación de la "mera moral". Anthropos Editorial.

Vinnari, E., and Laine, M. (2017). The moral mechanism of counter accounts: The case of industrial animal production. Accounting, Organizations and Society, 57, 1-17.

Vitell, S. J., and Singhapakdi, A. (2008). The role of ethics institutionalization in influencing organizational commitment, job satisfaction, and esprit de corps. Journal of Business Ethics, 81(2), 343-353.

Wells, C. (2014). Two eras of civic information and the evolving relationship between civil society organizations and young citizens. New Media \& Society, 16(4), 615-636.

Wijen, F. (2014). Means versus ends in opaque institutional fields: Trading off compliance and achievement in sustainability standard adoption. Academy of Management Review, 39(3), 302-323.

Wittgenstein, L. (1965). I: A lecture on ethics. The philosophical review, 74(1), 3-12. 
